\title{
Effect of Dezocine Combined with Dexmedetomidine on Postoperative Pain and Quality of Life in Patients with Advanced Liver Cancer
}

\author{
XIAOLAN LIU, Y. HE, XIAOXIA CAO, LINGHUI LIU AND H. TAN* \\ Department of Anesthesiology, The Central Hospital of EnshiTujia and Miao Autonomous Prefectrue, Enshi, China
}

Liu et al: : Effect Dezocine and Dexmedetomidine combination on Postoperative Pain

\begin{abstract}
To investigate the effect of dezocine injection combined with dexmedetomidine on pain and quality of life in patients with advanced hepatocellular carcinoma. 64 patients with advanced liver cancer undergone surgery in the Central Hospital of EnshiTujia and Miao Autonomous centre from January 2016 to January 2019 were selected and randomly assigned to the experimental group and the control group. The control group was anesthetized with remifentanil during the operation and the experimental group was given remifentanil along with a combination of dezocine combined with dexmedetomidine. After the anesthesia, the time of extubating and the recovery time of the 2 groups were monitored, the visual analogue scale was used to evaluate the degree of pain in both the groups of patients at 2,6 and $24 \mathrm{~h}$, respectively and the SF-36 dimensions of 7D before and after the surgery were scored for both the groups. The incidence of adverse reactions in both the groups of patients post-surgery were monitored and compared. The visual analogue scale scores of the experimental group were lower than those of the control group at 2,6 and $24 \mathrm{~h}$ after surgery $(p<0.05)$. The visual analogue scale scores at 6 and $24 \mathrm{~h}$ after surgery were statistically lower than those at $2 \mathrm{~h}$ after surgery. The extubation time, recovery time and orientation recovery time in the experimental group were all lower than those in the control group, the SF-36 dimension scores in both groups before the surgery were not significantly different. The scores of all dimensions in the experimental group were significantly higher than those in the control group post-surgery. The scores of physical function, mental function and life function significantly $(p<0.05)$ increased after intervention in both the groups. The incidence of adverse reactions was lower in the experimental group compared to the control group $(\mathbf{p}<\mathbf{0 . 0 5})$. Dezocine injection combined with dexmedetomidine could significantly relieve postoperative pain in patients with advanced hepatocellular carcinoma, shorten the recovery time and improve the quality of life.
\end{abstract}

Key words: Dezocine, dexmedetomidine, advanced liver cancer, pain, quality of life

Liver cancer refers to malignant tumor of liver and can be divided into two types, primary and secondary. Currently, the etiology of liver cancer is still unclear. It appears that the occurrence of this disease is influenced by many factors such as genetics, the environment and the life style. Statistical data showed that in 2012, the number of new cases of liver cancer in the world were 780000 accounting for $5.6 \%$ of the total number of cancers in the same period. In 2012, the number of new cases of liver cancer in China were 390000 accounting for $12.9 \%$ of the total number of cancers in the same period. The number of deaths were 380000 , accounting for about $17.4 \%$ of cancer deaths ${ }^{[1,2]}$. The above data points out that liver cancer has become a significant abnormality that threatens the lives and health of Chinese residents. At this stage, the treatment of liver cancer includes surgical treatment, chemotherapy and radiotherapy. Among them, surgical treatment is the first choice for liver cancer, and it is also the most effective means for treating it. By completely removing the lesion, it can significantly improve the patient's condition and its prognosis ${ }^{[3]}$. Remifentanil is a commonly used narcotic drug in the surgical treatment of liver cancer. It is mainly used for the induction and maintenance of general anesthesia. It has the advantages of greater efficacy and short maintenance time. However, clinical studies in recent years have found that remifentanil can cause hypersensitivity reactions and has the potential to reduce quality of life of patients ${ }^{[4,5]}$. The authors found that dezocine injection combined with dexmedetomidine could significantly relieve postoperative pain in patients undergoing

*Address for correspondence

E-mail: cpsturri@126.com 
advanced liver cancer surgery, shorten postoperative recovery time and ameliorate their quality of life.

Sixty four patients with advanced hepatocellular carcinoma who underwent surgery in the Central Hospital of EnshiTujia and Miao Autonomous Prefectrue from January 2016 to January 2019 were selected as the research subjects. They were randomly assigned to the experimental group and the control groups. Among the 32 patients in the experimental group, there were 18 males and 14 females in the age range of 43-67 y with an average age of $52.06 \pm 1.98 \mathrm{y}$. In this group 20 cases are in American Society of Anethesiologists physical status classification system (ASA) class I and 12 cases in class II. Among the 32 patients in the control group, 17 were males and 15 were females in the age range of $44-68 \mathrm{y}$ with an average age of $51.98 \pm 2.05 \mathrm{y}$ and in the group 19 cases are of ASA class I and 13 cases are class II. The general data of the two groups of patients such as gender, age, and anesthesia class was all similar and not statistically significant.

Inclusion criteria were, all those patients who were pathologically confirmed as advanced liver cancer concurrent surgical treatment, patients who were conscious and can cooperate with the investigation, those with complete medical records and those patients and their family members clearly understood the process, method, principle of the investigation and signed an informed consent form.

Exclusion criteria were, those with a combination of mental disorders, patients with other concurrent diseases such as coronary heart disease and renal failure; those with a combination of other malignant tumors and patients who are allergic to the use of narcotic drugs and sedatives.

After the two groups of patients were admitted to the hospital, they were exposed to some careful measures. Before the surgery $8 \mathrm{~h}$ fasting was implemented and $2 \mathrm{~h}$ prior to surgery water intake was prohibited. After the patients were transferred into the operating room, they were laid down for $5 \mathrm{~min}$ to establish a venous canula and then $5 \mathrm{mg} / \mathrm{ml}$ midazolam (YichangRenfu Pharmaceutical Co., Ltd., Chinese medicine quasi-word H20067040, 2011-07-08) was injected, remifentanil (Chinese Pharmaceutical Group Industry Co., Ltd.. Langfang Branch, Chinese medicine quasi-word $\mathrm{H}$ 20123421, 2012-12-31) and propofol (Xi'an Libang Pharmaceutical Co., Ltd., Chinese medicine quasiword H 20123318, 2012-11-20) was used to induced anesthesia, patients lost consciousness after tracheal intubation and maintaining anesthesia. Narcotic pump was used to infuse remifentanil and propofol, and the dose of vecuronium bromide was adjusted by the anesthesiologist according to the patient's tracheal muscle relaxing capacity. Finally, the patient in the experimental group was given $0.15 \mathrm{mg} / \mathrm{kg}$ of muscle relaxant (Yangzijiang Pharmaceutical Group Co., Ltd., Chinese medicine quasi-word H20080329, 2008-05-15) and infused with $1 \mu \mathrm{g} / \mathrm{kg}$ of dexmedetomidine (Jiangsu Hengrui Pharmaceutical Co., Ltd., Chinese medicine standard word H20090248, 2009-05-31), while patients in the control group were not administered any other drugs. Ten minutes before the end of the surgery propofol was stopped, at the end of the surgery remifentanil is also stopped, subsequently close monitoring of the patient's vital signs was done until the patient is awake after the antimicrobial treatment.

Visual analog scale (VAS) was used to assess the pain at 2, 6, and $24 \mathrm{~h}$ after surgery in both the groups of patients, VAS used a scale of $0-10$ in which, 1-4 is mild pain, 5-6 is moderate pain, 7-9 is severe pain, 10 for unbearable severe pain and the patient marks his own assessment of pain on the VAS ruler according to his own situation. This method requires higher abstract thinking of the patient and does not apply to elderly people or people with cognitive impairment ${ }^{[6]}$. A couple of times, the patient was asked by the health care provider to describe the intensity of pain for concrete and accurate results.

Responsible nurses recorded the post-operative extraction time, awakening time, and directional force recovery time of both groups of patients and compared them. The extraction time was from the end of the surgery to the patient's indication of pulling the tube, and the extraction of the tube was indicated from calling with the eyes to shaking the head ${ }^{[7]}$. The awakening time is from the end of the surgery until the patient's consciousness is completely restored. The patient can recall his name and the directional force recovery time is the patient's ability to accurately distinguish up and down the time.

Using the short form-36 item (SF-36) to evaluate the quality of life of the 2 groups of patients before and after surgery 7D, SF-36 is divided into 8 aspects such as physical functioning, bodily pain, general health, vitality, role emotional, mental health, physiological social functioning and emotional function. It can assess the quality of life of patients and has a wide range of clinical applications. The scale can be divided into 
physiological functions, psychological functions and life functions. The higher the score, the higher the quality of life ${ }^{[6]}$.

The incidence of adverse reactions such as dizziness, vomiting and low blood pressure were statistically compared between the 2 groups of patients after surgery. Small or medium doses of the drugs from both the categories can be administered to the patient targeting the pain receptors dually and minimizing the adverse effects resulting from toxic doses. Using SPSS220 to analyze the collected data, the counting data is expressed in the form of a rate (\%), the analysis is conducted using a card test, the measured data is expressed in the form (\%), the $\mathrm{T}$ test is used for analysis, and the difference of 0.05 was statistically significant.

After comparison, The VAS scores of the experimental group were significantly $(\mathrm{P}<0.05)$ lower than that of the control group at 2, 6 and $24 \mathrm{~h}$ after surgery and the VAS score at 6 and $24 \mathrm{~h}$ after surgery was significantly $(p<0.05)$ lower than that at $2 \mathrm{~h}$ after surgery as shown in Table 1. After the evaluation, the time of extraction,

TABLE 1: COMPARISON OF POSTOPERATIVE PAIN

\begin{tabular}{lcccc}
\hline \multirow{2}{*}{ Groups } & No of & \multicolumn{3}{c}{ VAS score post-surgery } \\
\cline { 3 - 5 } subjects & $2 \mathrm{~h}$ & $6 \mathrm{~h}$ & $24 \mathrm{~h}$ \\
Experimental & 32 & $6.12 \pm 0.11$ & $4.26 \pm 0.05^{*}$ & $2.09 \pm 0.15^{* \#}$ \\
group & & $7.26 \pm 0.12$ & $6.09 \pm 0.13^{*}$ & $3.61 \pm 0.34^{* \#}$ \\
Control group & 32 & 2.061 & 1.981 & 2.315 \\
$\mathrm{t}$ & - & $<0.05$ & $<0.05$ & $<0.05$ \\
$\mathrm{p}$ & - & $<$ &
\end{tabular}

TABLE 2: COMPARISON OF SURGICALINDICATORS BETWEEN THE TWO GROUPS

\begin{tabular}{lcccc}
\hline Group & $\begin{array}{c}\text { No of } \\
\text { subjects }\end{array}$ & $\begin{array}{c}\text { Extraction } \\
\text { time } \\
\text { (min) }\end{array}$ & $\begin{array}{c}\text { Wake } \\
\text { up time } \\
\text { (min) }\end{array}$ & $\begin{array}{c}\text { Orientation } \\
\text { recovery } \\
\text { time (min) }\end{array}$ \\
\hline $\begin{array}{l}\text { Experimental } \\
\text { group }\end{array}$ & 32 & $7.06 \pm 0.64$ & $7.34 \pm 0.81$ & $11.26 \pm 1.06$ \\
Control & 32 & $8.19 \pm 0.54$ & $9.08 \pm 0.12$ & $13.08 \pm 0.95$ \\
group & & 1.361 & 2.061 & 2.151 \\
t & - & $<0.05$ & $<0.05$ & $<0.05$ \\
P & - & $<$
\end{tabular}

awakening time and fixed force recovery time were significantly shorter in the experimental group than in the control group $(\mathrm{p}<0.05)$ as shown in Table 2.

The SF-36 dimension scores between the two groups before surgery were not significantly different. The SF-36 dimension score was significantly higher in the experimental group post-surgery compared to that of the control group $(p<0.05)$. After surgical intervention of the 2 groups of patients, the body function, psychological function and life function scores were all increased and the difference was statistically significant $(p<0.05)$, the specific data are as shown in Table 3. After evaluation, the incidence of postoperative adverse reaction was significantly lower than that of the control group in the experimental group $(\mathrm{p}<0.05)$ as shown in Table 4 .

In recent years, with the changes in the lifestyle and various types of chemical pollution in the environment, the incidence of liver cancer has increased year by year and statistical data shows that about 383000 people in China die of liver cancer, accounting for $51 \%$ of the global death toll ${ }^{[7-9]}$. Typical clinical symptoms of liver cancer include excruciating pain, fatigue, anorexia, jaundice and upper gastrointestinal bleeding. If timely treatment is not given to the patient, patients can develop associated complications like ascites, liver and kidney failure, which seriously impact morbidity and mortality of patients ${ }^{[10,11]}$. At this stage, surgery is the first choice of treatment for liver cancer. Through radical hepatectomy or palliative hepatectomy, it can significantly relieve the clinical symptoms of patients and improve their prognosis ${ }^{[12,13]}$. However, clinical practice has confirmed that patients with advanced liver cancer surgery would suffer significant pain, trigger their body stress response, and reduce the patient's immune function. Remifentanil is a $\mu$ opioid receptor agonist, which can reach the peak effect within a short period of time after administration. As Remifentanil is 100 times more potent than morphine, respiratory depression is far less observed than other narcotics. Moreover, the efficacy of this drug recedes faster and is therefore often used in anesthesia in advanced

TABLE 3: COMPARISON OF POSTOPERATIVE QUALITY OF LIFE SCORES

\begin{tabular}{lccccccc}
\hline \multirow{2}{*}{ Groups } & $\begin{array}{c}\text { No of } \\
\text { subjects }\end{array}$ & \multicolumn{2}{c}{ Physical function } & \multicolumn{2}{c}{ Psychological function } & \multicolumn{2}{c}{ Life function } \\
\cline { 3 - 8 } & & $\begin{array}{c}\text { Before } \\
\text { intervention }\end{array}$ & $\begin{array}{c}\text { After } \\
\text { intervention }\end{array}$ & $\begin{array}{c}\text { Before } \\
\text { intervention }\end{array}$ & $\begin{array}{c}\text { After } \\
\text { intervention }\end{array}$ & $\begin{array}{c}\text { Before } \\
\text { intervention }\end{array}$ & $\begin{array}{c}\text { After } \\
\text { intervention }\end{array}$ \\
Experimental & 32 & $53.97 \pm 3.29$ & $86.97 \pm 2.08^{*}$ & $60.96 \pm 1.53$ & $81.76 \pm 1.38^{*}$ & $56.39 \pm 2.34$ & $83.67 \pm 1.62^{*}$ \\
group & 32 & $54.09 \pm 2.98$ & $71.59 \pm 1.75^{*}$ & $61.98 \pm 0.59$ & $73.05 \pm 2.05^{*}$ & $55.96 \pm 1.98$ & $71.63 \pm 2.21^{*}$ \\
Control group & - & 0.681 & 1.638 & 0.721 & 2.367 & 0.813 & 1.961 \\
t & - & $>0.05$ & $<0.05$ & $>0.05$ & $<0.05$ & $>0.05$ & $<0.05$ \\
P & & & & & &
\end{tabular}


TABLE 4: COMPARISON OF THE INCIDENCE OF POSTOPERATIVE ADVERSE REACTIONS.

\begin{tabular}{lccccc}
\hline Group & Cases & Dizziness (\%) & Vomiting (\%) & Low BP (\%) & Incidence (\%) \\
\hline Experimental group & 32 & $2(6.25)$ & $1(3.13)$ & $1(3.13)$ & $4(12.5)$ \\
Control group & 32 & $3(9.38)$ & $3(9.38)$ & $1(3.13)$ & $7(21.88)$ \\
$\mathrm{t}$ & - & - & - & - & 4.634 \\
$\mathrm{P}$ & - & - & - & - & $<0.05$ \\
\hline
\end{tabular}

liver cancer surgery. However, some studies have pointed out that most narcotic drugs will cause pain and allergy in patients after application, which will not only increase postoperative pain in patients but also the incidence of postoperative restlessness and risk events in patients ${ }^{[14,15]}$. Dezocine possessed both agonist and antagonist activity as it is a k-receptor agonist and $\mu$ receptor antagonist. Its main physiological effects are analgesia, mild addiction and high efficacy. It is mainly used in the treatment of acute pain. Dexmedetomidine is a type of $\alpha 2$-epinephrine receptor agonist with sedative, analgesic and blocking nerve transmission effects. It is often used as a general anesthesia in patients. The study pointed out that the combination of the two can significantly reduce postoperative pain in patients with advanced liver cancer ${ }^{[16-18]}$.

In a study on 54 patients undergoing general anesthesia, the role of dezocine and dexmedetomidine in reducing allergic pain seen after anesthesia was investigated. The results showed that intraoperative hemodynamics of patients who were given a combination of dezocine and remifentanil was found to be relatively stable ${ }^{[18]}$. At the same time, the patient's postoperative pain degree and Ramsay score were better than those of the patients who simply used dezocine or dexmedetomidine alone. This study reported that dexmedetomidine was able to effectively inhibit the cough reaction during tubing. Dezocine could block the central nervous system impulse to reduce the central sensitization caused by tissue damage and thus act as a good analgesic. Another study reported similar result by administering different doses of dexmedetomidine to 120 patients with general anaesthesia in hysterectomy. The combined background dose of dezocine was $0.1 \mu \mathrm{g} / \mathrm{kg} / \mathrm{h}^{[19]}$. The analgesia was better and the patients reported low postoperative pain with a lower incidence of postoperative adverse reaction resulting in better comfort. Liao and Zhang reported that in a group of 90 patients the combined use of dezocine and dexmedetomidine for general anesthesia could stabilize the blood pressure and heart rate in patients and reduce the incidence of postoperative restlessness ${ }^{[20]}$. These authors speculated that this was related to dexmedetomidine inhibiting the release of norepinephrine.

Special Issue 3, 2020
In the present investigation through an experimental group and control group the effects of a combination of dezocine and dexmedetomidine on postoperative pain and quality of life was investigated in patients with advanced liver cancer under general anesthesia. The perceived pain at different timings in the experimental group that received dezocine and dexmedetomidine was lower than that of the control group using remifentanil and the resuscitation time, directional force recovery time and postoperative complication rate of the experimental group were lower than that of the control group. The post-surgery quality of life in the experimental group was better than that in the control group. The study speculated that this is because dexmedetomidine could inhibit the release of norepinephrine from the adrenergic nerve endings and reduce the level of catecholamine in the blood circulation through stimulating $\alpha$-2-epinephrine receptors. This effect therefore could stabilize the blood pressure and heart rate of patients during the postoperative awakening period, reduce the inhibition of the respiratory centre and at the same time, dezocine exerts a good analgesic and sedative effect, without any obvious inhibition of cardiovascular functioning. Dezocine could reduce pain by inhibiting nerve conduction in the central nervous system; therefore, the combined administration could reduce the pain of postoperative patients and achieve improvement in the quality of life.

All in all, dezocine injection combined with dexmedetomidine can significantly relieve postoperative pain in patients with advanced liver cancer surgery, shorten postoperative recovery time and improve their quality of life, and it is worth clinical promotion.

\section{Conflict of interest:}

The authors report no conflicts of interest in this work.

\section{REFERENCES}

1. Wei KJ, Peng XB, Liang ZH. Global Cancer Epidemic Overview. Chin Oncol 2015;24:621-30.

2. Guan CX, Wei WQ. Research progress in liver cancer prevention and treatment. J Liver Canc Elect 2015;2:42-7.

3. Yuan Y, Wang JY, Yuan F. Changes in the expression level of 
glycogen Synthetase kinase-3 $\beta$ in rats with hypersensitivity. Chin J Med 2012;92:435-9.

4. Guo YL. VAS score used in postoperative nursing of lumbar intervertebral disc herniation. Transport Med 2014;28:403-4.

5. Chen SG, Zhang ZQ. Discussion on the timing of tracheal extubation in patients with general anesthesia convalescence. $\mathrm{J}$ Clin Care 2012;11:45-6.

6. Yu JM, Sun H, Wu C, Dong CS, Lu Y, Zhang Y. The analgesic effect of ropivacaine combined with dexmedetomidine for incision infiltration after laparoscopic cholecystectomy. SurgLaparosEndosPercut Tech 2016;26:449-54.

7. Dong CS, Lu Y, Zhang J, Sun P, Yu JM, Wu C, et al. The optimal dose of dexmedetomidine added to a sufentanil-based analgesic regimen for postoperative pain control in spine surgery: A probit analysis study. Med 2016;95-39.

8. Huang GX, Chen D. Analysis of the effect of right metomidine and Shufentaini on hemorheology and stress response in patients with liver cancer. Hebei Med 2016;38:3118-20.

9. Cao JL, Pei YP, Wei JQ, Zhang YY. Effects of intraoperative dexmedetomidine with intravenous anesthesia on postoperative emergence agitation/delirium in pediatric patients undergoing tonsillectomy with or without adenoidectomy: A Consortprospective, randomized, controlled clinical trial. Med 2016;95:49.

10. Tian JM, Zheng WQ, Shen JB. Effect of Rimetomidine and Sevoflurane on the Cognitive Function of Patients with Aging Surgery. ContCardiovas Dis 2017;17:475-7.

11. Zhang WW, Zhang H, Cheng W. Effect of dextromethidine combined with dzosin on hypersensitivity in patients with retroperitoneal endoscopic surgery after Ruifentaini infusion. J Xuzhou Med Col 2016;36:709-12.

12. Zhang GQ, Hua L, Zhang GL. The analgesic effect of right metomidineFuheruifen after lung cancer resection and its effect on body immune function. Chin Pharm 2016;27:4215-7.

13. Nishimura LT, Auckburally A, Santilli J, Vieira BH, Garcia
DO, Honsho CS, et al. Effects of dexmedetomidine combined with commonly administered opioids on clinical variables in dogs. Am JVeter Res 2018;79:267-75.

14. Jin JX, Wang Y, Pan MZ. Comparison of different doses of right metomide combined with Xinkaifen in patients with controlled intravenous analgesia after total hysterectomy. Chin Sex Sci 2016;25:44-7.

15. Xie ZR, Luo YL, Xiao FM. Personalized nursing care for liver dissociation and portal vein ligation in liver cancer patients. $\mathrm{J}$ Integ Modern Chin West Med 2016;25:4096-8.

16. Lv GS, Chen L, Wang HY. Present situation and prospect of liver cancer research in China. Life Sci 2015;27:237-48.

17. Ma SS. Clinical Observation on the Prevention of Rifentanyl Pain Allergy with MarzanneParacisb Sodium. Chin J Phys 2012;14:549-51.

18. Wan YL, Chen XY, Lin T. The effect of right-metomidine combined with dizosin on hemodynamics and analgesic sedation during thoracic surgery. Chin Pharm 2013;24:3616-8.

19. Yu XC. The application of diazocin combined with different doses of right metomidine in postoperative intravenous analgesia. Tianjin Med 2013;41:917-8.

20. Liao Q, Zhang $\mathrm{H}$. The effects of right-metomidine and dizosin on restlessness and hemodynamics in patients with general anesthesia during rousing period. Hebei Med 2015;21:389-92.

This is an open access article distributed under the terms of the Creative Commons Attribution-NonCommercial-ShareAlike 3.0 License, which allows others to remix, tweak, and build upon the work non-commercially, as long as the author is credited and the new creations are licensed under the identical terms

This article was originally published in a special issue, "Biomedical research applications in Pharmaceutical Sciences" Indian J Pharm Sci 2020:82(2)spl issue3;36-40 